\title{
Optical beam steering based on electromagnetically induced transparency
}

\author{
Qingqing Sun, Yuri V. Rostovtsev, and M. Suhail Zubairy \\ Department of Physics and Institute of Quantum Studies, Texas A\&M University, College Station, Texas 77843-4242, USA
}

(Received 9 December 2005; published 26 September 2006)

\begin{abstract}
We propose a scheme that provides all-optically-controlled steering of light beam. The system is based on steep dispersion of a coherently driven medium. Using the eikonal equation, we study the steering angle, the spread of the optical beam, and the limits set by residual absorption of the medium under conditions of electromagnetically induced transparency. Implementation of another scheme for ultrashort pulses is also discussed.
\end{abstract}

DOI: 10.1103/PhysRevA.74.033819

PACS number(s): 42.50.Gy, 42.50.Nn

\section{INTRODUCTION}

Optical beam deflection is an important technology in modern optics. It has applications in the fields of radar, optical imaging, laser machining, and free space communication. Many physical mechanisms have been used to obtain the deflection $[1,2]$. Among them mechanical motion [3-5] is the simplest and most convenient way, since it only mechanically moves or rotates the deflector (mirror, grating, etc.). A thermal gradient [6], acousto-optical interaction [7-9], and the electro-optic effect [10-12] all can induce a refractive index gradient which deflects the light. The electro-optic deflectors are faster than their acousto-optic counterparts as compared in Ref. [13]. Nowadays with the development of new materials and devices, attention has been focused on using photonic crystals $[14,15]$ and phased arrays $[16-18]$ to get fast beam steering. For a recent review of electro-optical systems, see Ref. [19].

Light can also change the propagation direction of another beam of light through interaction with matter. Beam deflections have been reported in sodium vapor via optical pumping [20] and in rubidium vapor via saturated absorption and hyperfine pumping [21]. Electromagnetically induced transparency (EIT) provides another mechanism since the refractive index can change near the transparency center [22-24]. Moseley et al. first observed the electromagnetically induced focusing and defocusing effects in a rubidium vapor $[25,26]$, which come from the spatial Gaussian distribution of the pump field. These authors included both refraction and absorption modification in the numerical calculation and found a qualitative accordance with experiment. Mitsunaga et al. observed absorption imaging in cold sodium atoms [27]. The probe is mostly absorbed after the atom cloud except for the focal point of the pump beam. Transmission through this EIT point can reach almost $200 \%$, which is obviously a focusing feature. Another example based on EIT is electromagnetically induced waveguiding [28-30] which uses the driving field as a fiber to confine the probe field. Raman spatial solitons have been demonstrated by Walker et al. [31-33]. As an alternative to the near-resonant configuration used in the above papers, the pump and probe can both be detuned to the blue side and remain in two-photon resonance. Induced focusing and waveguiding in such a $\Lambda$ system have been discussed under EIT [34] and coherent population trapping (CPT) conditions [35]. Shpaisman et al. [36] have discussed both these cases and have extended the method to double- $\Lambda$ systems, where one $\Lambda$ system can induce waveguiding in the other. The maximum Rabi frequency of the pump was chosen near the EIT or CPT threshold to ensure low absorption and significant focusing.

In this paper we explore the possibilities and limits of beam deflection through the EIT effect. An inhomogeneous pump field intensity produces a refractive index gradient for the probe. Ray optics is adopted to analyze the steering angle and absorption for each probe ray. Under optimal pump distribution rays of the same frequency can be deflected at the same angle, unaffected by the starting position. For a single frequency we can also obtain exact focusing. Finally we show that even a whole beam with spatial and spectral width can be deflected together using a specific setup.

\section{BEAM PROPAGATION IN AN INHOMOGENEOUS MEDIUM}

The idea of all-optical steering of an electromagnetic wave is as follows. Consider a pulse of central frequency $\omega$ propagating through a three-level EIT medium as shown in Fig. 1. We assume that the spectral width of the pulse lies well within the EIT window such that the inequalities $\Omega \gamma$, $\gg \Delta \omega$ are satisfied. Here $\Omega$ is the Rabi frequency of the driving field resonant with an $a-c$ transition, $\gamma$ is the atomic decay rate, and $\Delta \omega=\omega-\omega_{a b}$ is the detuning of the probe frequency $\omega$ with the atomic transition $a-b$. Now in order to steer the incident pulse in a different direction, we introduce a phase shift for the different transverse positions $x$ of the pulse at the output by modulating the Rabi frequency of the driving field. Thus the whole pulse has a different direction while coming out of the EIT medium. Here we derive a simple expression for the beam steering angle and the corresponding losses.

We assume that we have a highly dispersive medium. For a large enough $\Omega$, the index of refraction for the probe field can be written as

$$
n^{\prime} \simeq 1+\frac{\chi^{\prime}}{2} \simeq 1+\alpha \Delta \omega,
$$

where $N$ is the atomic density and $\alpha=N|\wp|^{2} / 2 \varepsilon_{0} \hbar \Omega^{2}$ with $\wp$ being the atomic dipole moment on the $a-b$ transition. Inhomogeneous $\Omega(x)$ leads to inhomogeneous $n^{\prime}(x)$. 
(a)

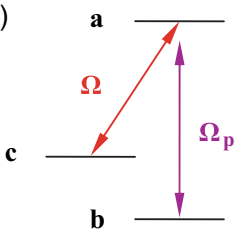

(c)

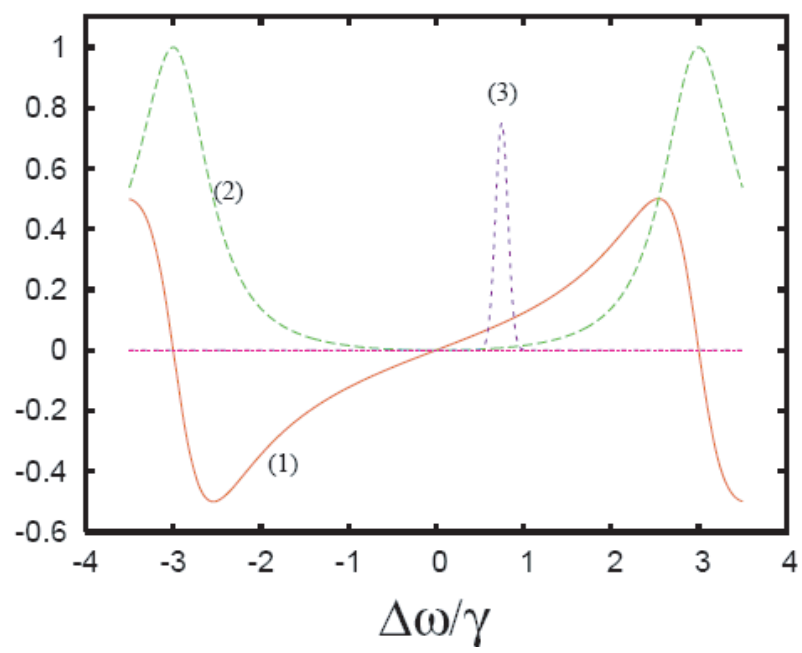

FIG. 1. (Color online) (a) The schematics of the three-level atomic system with the fields. (b) A slab of the three-level atomic medium turns the probe light via an inhomogeneous driving field. (c) The real (1) and imaginary (2) parts of the atomic susceptibility, and the spectrum of probe pulse (3) vs probe frequency.

The trajectory of the light rays propagating in an inhomogeneous medium can be found by using the eikonal approximation [37]. We start with Maxwell's equation, which describes the propagation of the electromagnetic waves,

$$
\nabla^{2} E-\frac{1}{c^{2}} \frac{\partial^{2} E}{\partial t^{2}}=\mu_{0} \frac{\partial^{2} P}{\partial t^{2}} .
$$

We can expand the field and the polarization in terms of the slowly varying amplitudes $E_{\nu}$ and $P_{\nu}$ and the eikonal $\psi$ as

$$
E=\sum_{\nu} E_{\nu} e^{-i \nu t+i k \psi}, \quad P=\sum_{\nu} P_{\nu} e^{-i \nu t+i k \psi} .
$$

Here $k=\nu / c$. The polarization of the medium is related to the field intensity as $P_{\nu}=\varepsilon_{0} \chi_{\nu} E_{\nu}$, where the susceptibility is $\chi_{\nu}$ $=\chi_{\nu}^{\prime}+i \chi_{\nu}^{\prime \prime}$. If we neglect the second-order derivative over coordinates for the amplitude $E_{\nu}$, the eikonal equation is given by

$$
(\nabla \psi)^{2}=1+\chi_{\nu}^{\prime} \simeq n^{\prime 2} .
$$

So we can write down $\nabla \psi=n^{\prime} d \mathbf{R} / d s$ and obtain the geometrical optics differential equation in the vector form

$$
\frac{d}{d s}\left(n^{\prime} \frac{d \mathbf{R}}{d s}\right)=\nabla n^{\prime},
$$

where $\mathbf{R}$ is the point of the ray. Here $\mathbf{R}(x, z)=X(z) \hat{\mathbf{x}}+z \hat{\mathbf{z}}$ and $\hat{\mathbf{x}}, \hat{\mathbf{z}}$ are unit vectors along the axes. Then, for the $x$ and $z$ components,

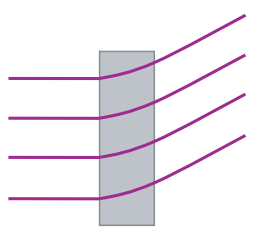

(a)
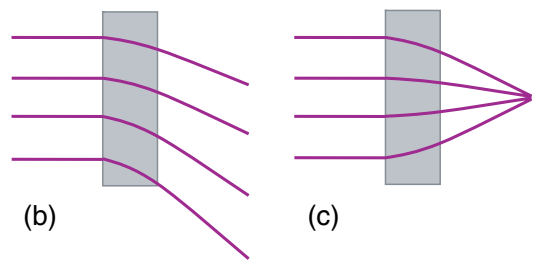

(c)
FIG. 2. (Color online) For different distributions of the driving field, different regimes of probe light propagation take place. (a) The light deflection angle is the same for all the light rays; (b) the probe light turns but spreads; (c) focusing of the probe.

$$
\frac{d}{d s}\left(n^{\prime} \frac{d X}{d s}\right)=\frac{\partial n^{\prime}}{\partial x} \text { and } \quad \frac{d}{d s}\left(n^{\prime} \frac{d z}{d s}\right)=\frac{\partial n^{\prime}}{\partial z} .
$$

The equation describing the amplitude of the electromagnetic field can be obtained in a similar manner. It follows from the imaginary part of Eq. (2) that

$$
2 k \nabla \psi \nabla E_{\nu}+k\left(\nabla^{2} \psi\right) E_{\nu}=-\frac{\nu^{2}}{c^{2}} \chi^{\prime \prime} E_{\nu}
$$

The solution of the above equation has the following form

$$
E_{\nu}=\frac{E_{0 \nu}}{\sqrt{n^{\prime}}} \exp \left(-\int_{s_{1}}^{s_{2}} \frac{\nu \chi_{\nu}^{\prime \prime}}{2 n^{\prime} c} d s\right) .
$$

In the next section we will discuss several inhomogeneous driving field distributions and their steering effects.

\section{DISCUSSION}

\section{A. Single-frequency deflection}

For the first case assume that

$$
\Omega(x)=\Omega_{0} / \sqrt{1+\beta x},
$$

where $\beta$ is the parameter that determines the inhomogeneous distribution of the driving field. The refractive index is therefore of the form $n^{\prime}(x) \simeq 1+\alpha_{0} \Delta \omega(1+\beta x)$ with $\alpha_{0}$ $=N|\wp|^{2} / 2 \varepsilon_{0} \hbar \Omega_{0}^{2}$. This form leads to constant $\nabla n^{\prime}$ for a single frequency. In the following we show that, for such a situation, we obtain the same steering angle for all the transverse positions $x$ as shown in Fig. 2(a). If the dependence of the driving field has a different form then there will be a spread of the optical rays as shown in Fig. 2(b). In Eq. (6) using $d s=\sqrt{d X(z)^{2}+d z^{2}}$ and $n^{\prime}=1+\alpha_{0} \Delta \omega(1+\beta x)$, we obtain

$$
\frac{d}{d s}\left(n^{\prime} \frac{d X}{d s}\right)=\alpha_{0} \Delta \omega \beta \text { and } \frac{d}{d s}\left(n^{\prime} \frac{d z}{d s}\right)=0 .
$$

The ordinary differential equation to describe the ray trajectory is given by

$$
\frac{d^{2} X(z)}{d z^{2}}=\frac{1+\alpha_{0} \Delta \omega[1+\beta X(z)]}{\left[1+\alpha_{0} \Delta \omega\left(1+\beta X_{0}\right)\right]^{2}} \alpha_{0} \Delta \omega \beta \simeq \alpha_{0} \Delta \omega \beta,
$$

and its solution is 

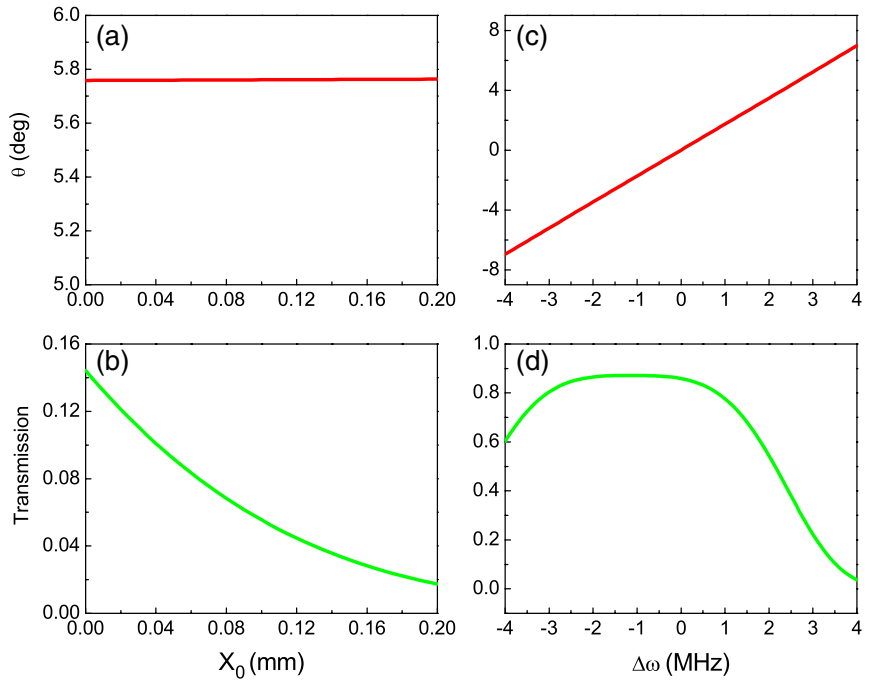

FIG. 3. (Color online) Deflection angle and transmission $\left|E_{\nu} / E_{0 \nu}\right|^{2}$ for $\Omega(x)=\Omega_{0} / \sqrt{1+\beta x}$. In (a), (b) the detuning is fixed at $\Delta \omega=3.3 \mathrm{MHz}$; the angle is constant when $X_{0}$ varies from 0 to $0.2 \mathrm{~mm}$. In (c), (d) $X_{0}=0$ while $\Delta \omega$ changes from -4 to $4 \mathrm{MHz}$; the angle follows the detuning linearly.

$$
X(z) \simeq X_{0}+\frac{\alpha_{0} \Delta \omega \beta z^{2}}{2} .
$$

The light turning angle $\theta$ can be found from $d X(z) / d z$ $=\tan \theta \simeq \theta$. The resulting angle is

$$
\theta \simeq \alpha_{0} \Delta \omega \beta L=\frac{N|\wp|^{2}}{2 \varepsilon_{0} \hbar \Omega_{0}^{2}} \Delta \omega \beta L .
$$

A calculation based on the full expression of $\chi$ [38] shows that these simple estimations Eqs. (12) and (13) are valid for small deflections. With the parameters $\beta=5000 \mathrm{~m}^{-1}, L$ $=1 \mathrm{~cm}, \Omega_{0}=50 \pi \mathrm{MHz}, N=10^{15} \mathrm{~cm}^{-3}, \lambda=0.5 \mu \mathrm{m}, \gamma_{a b}=\gamma_{\mathrm{rad}}$ $=2 \pi \mathrm{MHz}, \gamma_{c b}=1 \mathrm{kHz}$ we get Fig. 3. A probe beam with detuning $\Delta \omega=3.3 \mathrm{MHz}$ [Figs. 3(a) and 3(b)] will experience the same deflection angle $5.76^{\circ}$, although the ray starting position $X_{0}$ ranges from 0 to $0.2 \mathrm{~mm}$. The transmission decreases at larger $X_{0}$ because there $\Omega$ is smaller. Figures 3(c) and $3(\mathrm{~d})$ show the linear dependence of the deflection angle on detuning. Now $X_{0}$ is fixed at 0 and $\Delta \omega$ varies from -4 to $4 \mathrm{MHz}$. The transmission at negative $\Delta \omega$ is larger because the ray goes to the negative $x$ direction where $\Omega$ is larger. In this paper we have assumed the medium to be homogeneously broadened. For an inhomogeneously broadened medium the transmission would be many orders smaller. However, the steering angle could still be the same. Another factor that influences the transmission is $\gamma_{c b}$. This decay rate can be much larger under high density. If $\gamma_{c b}$ $=10^{4} \mathrm{~Hz}$ then the transmission will be ten times smaller while the angle will remain unaffected. On further increasing $\gamma_{c b}$ the transmission will decrease much faster.

To study the behavior of a beam with a finite diameter we perform some numerical simulation. Consider a monochromatic electromagnetic wave with frequency $\omega$; the Helmholtz equation is given by

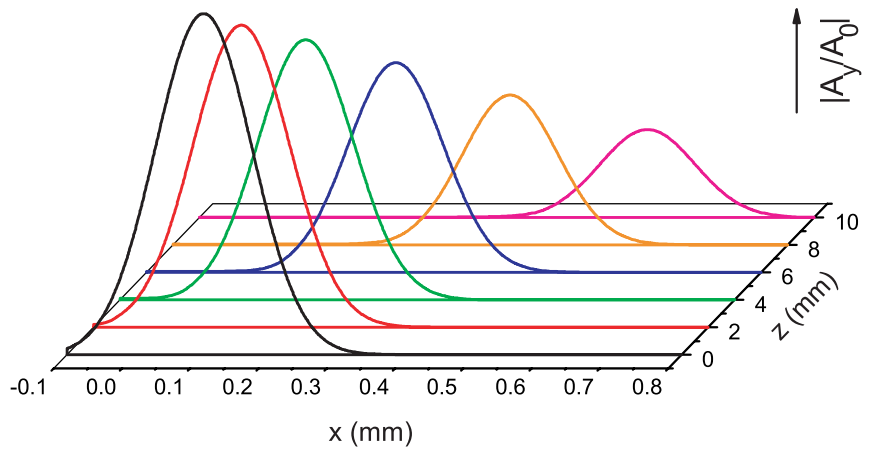

FIG. 4. (Color online) Numerical simulation for beam propagation. Here the detuning $\Delta \omega=3.3 \mathrm{MHz}$.

$$
\nabla^{2} \mathbf{E}+2 \boldsymbol{\nabla}[\mathbf{E} \cdot \nabla(\ln n)]+\frac{\omega^{2}}{c^{2}} n^{2} \mathbf{E}=\mathbf{0}
$$

where $n=n^{\prime}+i n^{\prime \prime}=\sqrt{1+\chi}$. For this inhomogeneous medium, $n_{0}=1$ and $\delta n(\mathbf{r})=n(\mathbf{r})-n_{0}, \quad k_{0}=\omega n_{0} / c, \quad \mathbf{r}=\left(\mathbf{r}_{\perp}, z\right), \quad \mathbf{E}(\mathbf{r}, t)$ $=\exp \left(i k_{0} z-i \omega t\right) \mathbf{A}\left(\mathbf{r}_{\perp}, z\right)$. We assume that the field has linear polarization set to the $y$ direction, and at the entrance it is given by

$$
\mathbf{A}(x, 0)=A_{0} \exp \left[-\left(\frac{x-x_{0}}{w_{0}}\right)^{2}\right] \hat{y}
$$

where $w_{0}=0.1 \mathrm{~mm}$ is the beam waist. $x_{0}=0.1 \mathrm{~mm}$ is the beam center at the entrance.

To get an accurate result we go beyond the paraxial limit, following the method of Refs. [39,40]. Separating the field into transverse and longitudinal components and expanding the equation in the small parameters $\lambda / w_{0}$ and $\delta n$, we obtain

$$
\begin{aligned}
i \frac{\partial A_{y}}{\partial z}= & \frac{1}{2 n_{0} k_{0}}\left(\frac{\partial^{2} \delta n}{\partial x^{2}} A_{y}+2 \frac{\partial \delta n}{\partial x} \frac{\partial A_{y}}{\partial x}+\delta n \frac{\partial^{2} A_{y}}{\partial x^{2}}\right)-\frac{1}{2 k_{0}} \frac{\partial^{2} A_{y}}{\partial x^{2}} \\
& -\frac{k_{0} \delta n}{n_{0}} A_{y} .
\end{aligned}
$$

It is then straightforward to get the beam distribution at $z$ $>0$. To compare with the result in Figs. 3(a) and 3(b) we use the same parameters. The only difference is now it is a beam instead of a ray. The simulation result is shown in Fig. 4. It clearly shows the beam being deflected and absorbed as it propagates along the $z$ axis. The peak of the beam goes to $X_{1}=0.556 \mathrm{~mm}$. This is a little smaller than the value $X_{1}$ $=0.600 \mathrm{~mm}$ of a ray starting from $X_{0}=0.1 \mathrm{~mm}$, which is reasonable since the absorption increases with $x$ and thus lowers the peak position. The peak intensity of the beam is $\left|A_{y} / A_{0}\right|^{2}=0.0664$, slightly higher than the ray transmission 0.0555 . The deflection angle from the simulation is $5.75^{\circ}$, matching the former result precisely. This angle is almost a constant for the whole beam despite its extremely slow increase with $z$.

From this example we see that the deflection angle could be as large as 


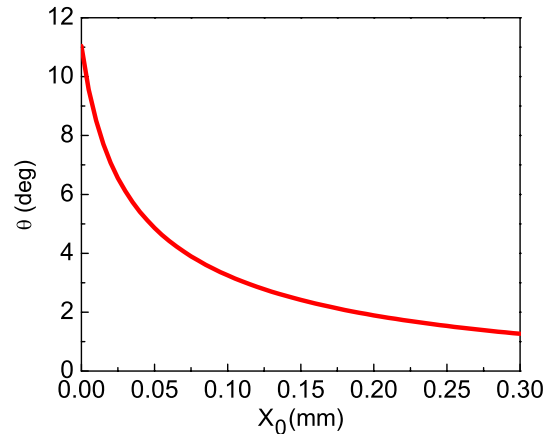

FIG. 5. (Color online) Deflection angle for $\Omega(x)=\Omega_{0}(1+\beta x)$. The detuning is fixed at $\Delta \omega=3.3 \mathrm{MHz}$. The angle is not a constant for varying $X_{0}$.

$$
\theta \simeq 0.1 \mathrm{rad}
$$

with affordable losses, which shows a potential for alloptical light steering. Here the deflection angle is only constant for a single frequency, but this is not a big problem for a probe pulse with narrow bandwidth.

As we have mentioned before, other driving field distributions will lead to spread of the probe pulse because the refractive index gradient depends on the spatial coordinates [see Fig. 2(b)]. Consider the simplest case $\Omega(x)=\Omega_{0}(1$ $+\beta x)$, which gives $n^{\prime}(x)=1+\alpha_{0} \Delta \omega /(1+\beta x)^{2} . \beta=1500 \mathrm{~m}^{-1}$ and all the other parameters are the same as in the first case. Now probe rays with $\Delta \omega=3.3 \mathrm{MHz}$ starting from different $X_{0}$ have different deflection angles, as shown in Fig. 5. There is spreading even for a single frequency, so this is not suitable for beam steering.

\section{B. Focusing and defocusing}

Next we show that, by controlling the spatial dependence of the Rabi frequency of the driving field, we can have a focusing or defocusing coherent medium, which adds additional flexibility to handle the probe field. Thus the coherent medium not only can act as an effective beam deflector but also can be transformed into a lens with controllable focal distance. We recall that both Moseley et al. [25] and Mitsunaga et al. [27] are using Gaussian coupling beams so the focusing is not very intense. Here we assume that the Rabi frequency of the driving field depends on the transverse coordinate via

$$
\Omega(x)=\frac{\Omega_{0}}{\sqrt{1-\beta x^{2}}} .
$$

The space-dependent refractive index is then given by $n^{\prime}(x)=1+\alpha_{0} \Delta \omega\left(1-\beta x^{2}\right)$. This creates the ray structure shown in Fig. 2(c). To see this, we consider a ray that starts at $X_{0}$. As a simple estimate, it goes out of the cell at $X_{1}$ $=X(L) \simeq X_{0}\left(1-\alpha_{0} \Delta \omega \beta L^{2}\right)$ and the deflection angle is $\theta \simeq$ $-2 \alpha_{0} \Delta \omega \beta L X_{0}$. This ray passes the axis at the distance
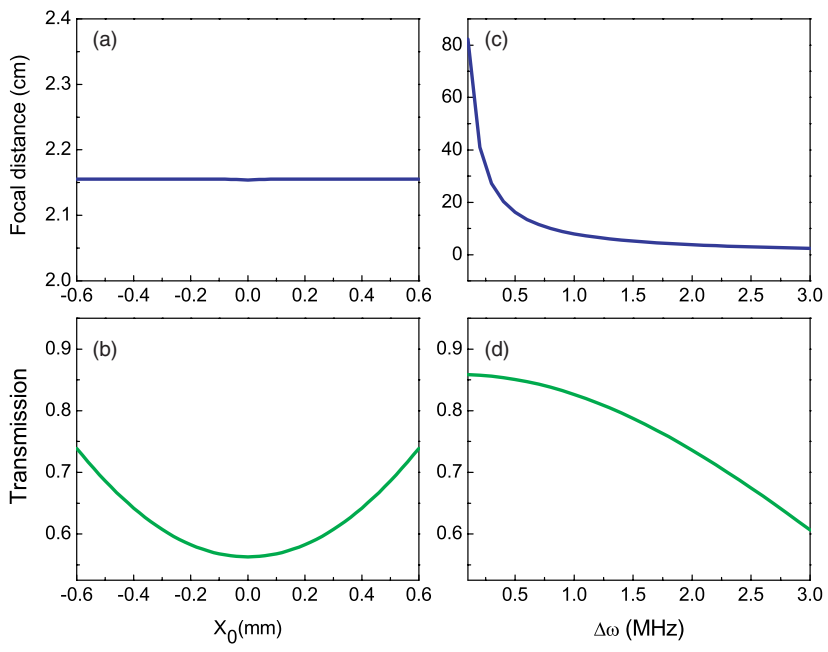

FIG. 6. (Color online) Focusing effect for $\Omega(x)=\Omega_{0} / \sqrt{1-\beta x^{2}}$. In (a), (b) the detuning is fixed at $\Delta \omega=3.3 \mathrm{MHz}$; the focal distance is constant when $X_{0}$ varies from -0.6 to $0.6 \mathrm{~mm}$. In (c), (d) $X_{0}=0$ while $\Delta \omega$ changes from 0.1 to $3 \mathrm{MHz}$; the angle follows Eq. (19).

$$
F=\frac{X_{1}}{-\theta} \simeq \frac{1-\alpha_{0} \Delta \omega \beta L^{2}}{2 \alpha_{0} \Delta \omega \beta L},
$$

which is independent of the initial position of the ray, $X_{0}$. This represents a lens with a focal distance $F$.

A calculation based on the full expression also supports these estimates. Consider a system with $\Delta \omega=3.3 \mathrm{MHz}, \beta$ $=10^{6} \mathrm{~m}^{-2}$ and all the other parameters the same as in the first case. It is obvious from Fig. 6(a) that $F$ does not change after $X_{0}$. So the focal distance $F$ is well defined for a single frequency. However, this distance changes for different frequencies [see Fig. 6(c)] as in Eq. (19). It decreases for large detuning which is easy to understand since large detuning has a rapid refractive index change and the beam deflects quickly. The focal distance $F$ can also be controlled by varying $\Omega_{0}$ or $\beta$. Both smaller $\Omega_{0}$ and larger $\beta$ can lead to smaller focal distance. The reason is the same as above. Finally, by using a negative $\beta$ or negative $\Delta \omega$ we can obtain good defocusing effect. If both $\beta$ and $\Delta \omega$ are negative we get focusing again. This is more applicable for experiment since the highest driving field required is $\Omega_{0}$ at the center. Note that here the driving field depends on $x$ so this is only a two-dimensional focusing. To simulate a real lens it should depend on $r_{\perp}$.

\section{Short-pulse deflection}

Up to now our discussion applies to the propagation of continuous waves and pulses that have time duration long enough to fit the EIT window, i.e., $\Omega^{2} T / \gamma>1$, where $T$ is the pulse width. The problem with shorter pulses is that they have a broad spectrum that may not fit the EIT window, leading to substantial absorption and a nonlinear dispersion. As a result, we may encounter strong reshaping and absorption while the pulse propagates through the EIT medium.

Recently, we proposed a solution to the problem of broadband pulse propagation through an EIT medium [41]. The 


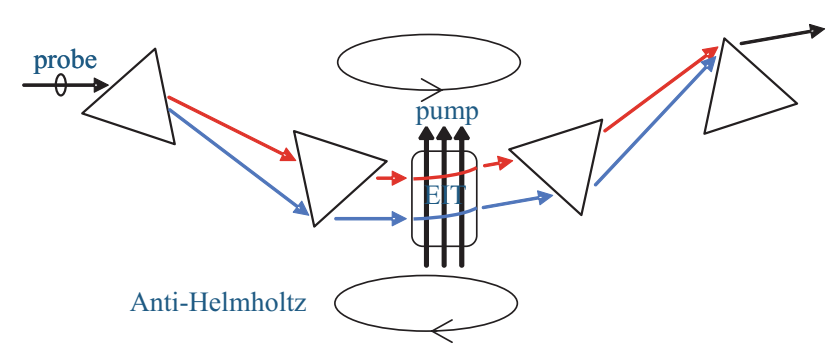

FIG. 7. (Color online) A scheme of optical steering for broadband pulses using a magnetic field gradient created by a pair of anti-Helmholtz coils.

main idea is that we can extend the absorptionless linear dispersion of the EIT three-level system using an inhomogeneous medium where we maintain the resonance with the optical field by shifting the atomic level via an inhomogeneous magnetic field. In our scheme, we have a gradient of the index of refraction due to the gradient of the magnetic field, because the detuning is position dependent.

The scheme is depicted in Fig. 7. The system consists of a set of prisms (or diffraction gratings) with total dispersion equal to zero. The first prism disperses the probe pulse into a divergent beam. The second prism transforms the beam into a parallel beam, with different frequencies shifted in space. When the beam goes into the EIT cell with cold atoms, an inhomogeneous magnetic field moves the level $b$ only so that each frequency is resonant with the local $a \leftrightarrow b$ transition. At the same time, the constant driving field propagates along the $x$ direction, which is resonant with the $a \leftrightarrow c$ transition.

Now a single-frequency ray at different $x$ positions will experience different detunings and refractive indexes because $\omega_{a b}$ changes with $x$ due to the applied inhomogeneous magnetic field. Such a refractive index gradient will cause deflection. For an ideal system, the frequency distribution after the second prism is linear, i.e., $d \omega / d x$ is a constant. Also $d \omega_{a b} / d x$ should be the same to match the field. We assume that a ray of frequency $\omega$ enters the EIT cell at $x_{0}$ position, and define $\delta x=x-x_{c}$ where $x_{c}$ is the position at which the ray is resonant, i.e., $\omega=\omega_{a b}\left(x_{c}\right)$. So the detuning at position $x$ is

$$
\Delta \omega(\delta x)=\omega-\omega_{a b}(x)=\omega_{a b}\left(x_{c}\right)-\omega_{a b}(x)=-\delta x \frac{d \omega_{a b}}{d x} .
$$

The refractive index will be

$$
n_{\omega}^{\prime}(\delta x) \simeq 1+\alpha_{0} \Delta \omega(\delta x)=1-\alpha_{0} \delta x \frac{d \omega}{d x} .
$$

The refractive gradient $\nabla n^{\prime}=-\alpha_{0}(d \omega / d x) \hat{\mathbf{x}}$ is a constant. It follows from Eqs. (12) and (13) that $\delta x_{1}-\delta x_{0}$ $\simeq\left|\nabla n^{\prime}\right| L^{2} / 2$ and $\sin \theta \simeq\left|\nabla n^{\prime}\right| L$ for small deflection. They do not depend on $\delta x_{0}$ or $\omega$. So all the rays go through parallel paths. This is crucial for the recombination of the pulse. Thus a probe beam with finite bandwidth and finite diameter can be deflected perfectly.
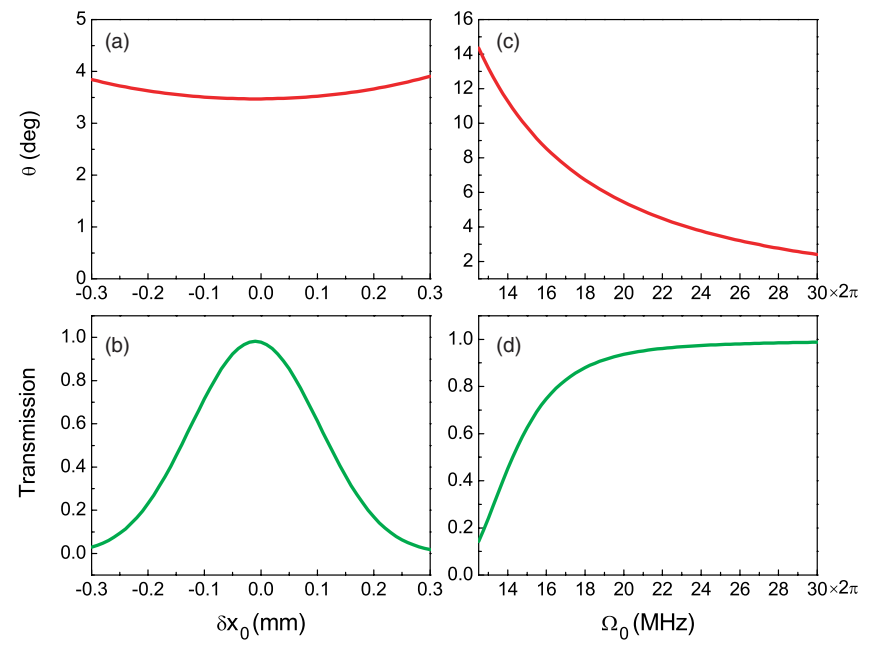

FIG. 8. (Color online) Dependence of the light deflection angle (a), (c) and the probe transmission (b), (d) on the position of the probe ray, $\delta x_{0}$, and on the magnitude of the Rabi frequency.

The above is only a simple estimate. In Fig. 8 we show some numerical results for $d \omega / d x=-10^{11} \mathrm{~Hz} / \mathrm{m}, L=1 \mathrm{~mm}$ and all the other parameters the same as in the first case. In Figs. 8(a) and 8(b) we can see the rays of frequency $2 \pi$ $\times 6 \times 10^{14} \mathrm{~Hz}$ starting from different $\delta x_{0}$. The deflection angles are around $3.47^{\circ}$ although a little larger for nonzero $\delta x_{0}$. The transmission for $\delta x_{0}=0$ is the highest $(0.98)$ but it decreases quickly for other starting positions. This is good enough since we can always put the central rays, which are the main part for an ordinary beam, at $\delta x_{0}=0$. Unlike the first case, now the frequency does not influence the deflection angle. A calculation for $\omega$ varying by $10^{10} \mathrm{~Hz}$ still gives the same curve, which is reasonable because only local decays are slightly changed. As a result the whole beam, despite its diameter and bandwidth, deflects at the same angle. If the diameter is small enough to fit within $\delta x_{0}= \pm 0.05 \mathrm{~mm}$, the whole pulse will be deflected with transmission $\sim 1$.

We can control the angle by varying the drive Rabi frequency, as shown in Figs. 8(c) and 8(d). The parameters are still the same, $\lambda=5000 \AA, \delta x_{0}=0$, only $\Omega$ varying from $2 \pi$ $\times 1.25 \times 10^{7} \mathrm{~Hz}$ to $2 \pi \times 3 \times 10^{7} \mathrm{~Hz}$. It is easy to find from the graph that a larger deflection angle accompanies smaller transmission. Angles greater than $10^{\circ}$ are even achievable at small driving field. But then the side rays (nonzero $\delta x_{0}$ ) suffer substantial absorption. For large driving field the system goes to the limit angle $\theta \rightarrow 0$ and transmission $\rightarrow 1$.

Since the level $b$ can be moved by a magnetic field, it must be a magnetic sublevel with $M \neq 0$ and have some nondegenerate magnetic sublevels nearby. If we include them in the calculation, the population in level $b$ will decrease, which effectively decreases the optical density. The residual absorption due to these off-resonance levels can be avoided by using a circularly polarized probe to interact with only level $b$.

\section{CONCLUSION}

We have shown that for a single-frequency or a narrowbandwidth probe field, high-quality deflection and focusing 
can be achieved under optimal pump distribution. The practical difficulty is how to generate such a distribution. One possible way is to put a screen with the desired transmission function as in Eqs. (9) and (18) behind the driving field. Pump diffraction may not be so severe if the propagation distance is short. Then we can control the deflection angle or focal distance by varying only the input driving field intensity $\left(\propto \Omega_{0}\right)$. This method is convenient and continuous. However, the scan speed is limited by the EIT establishment time, which is about the radiative lifetime $1 / \gamma_{\mathrm{rad}}$.

A short pulse can also be deflected to the same angle in the proposed scheme. The key point is here that the magnetic gradient provides the same refractive index gradient for all the frequency components. The deflection angle can also be controlled by the driving intensity. This method is promising due to its broadband ability. Here the problem is that the lowest-frequency gradient of a real prism system is
$10^{14} \mathrm{~Hz} / \mathrm{m}$ and it is only approximately constant. We need a better system to provide larger spatial dispersion.

In both of the deflection schemes the maximum deflection angle without significant absorption is $\sim 0.1 \mathrm{rad}$. Larger deflection always comes with larger absorption. This is determined by the relation between refractive index and absorption coefficient in EIT. Note that $0.1 \mathrm{rad}$ is already good enough for some applications, and additional devices like multiple birefringent prisms or holographic glass with multiple holograms can further increase the angle.

\section{ACKNOWLEDGMENTS}

We gratefully acknowledge the support from the Air Force Office of Scientific Research.
[1] V. J. Fowler and J. Schlafer, Proc. IEEE 54, 1437 (1966).

[2] M. Gottlieb, C. L. M. Ireland, and J. M. Ley, Electro-Optic and Acousto-Optic Scanning and Deflection (Dekker, New York, 1983).

[3] I. Cindrich, Appl. Opt. 6, 1531 (1967).

[4] D. H. McMahon, A. R. Franklin, and J. B. Thaxter, Appl. Opt. 8, 399 (1969).

[5] P. J. Brosens, Electro-Opt. Syst. Des. 3, 21 (1971).

[6] W. B. Jackson, N. M. Amer, A. C. Boccara, and D. Fournier, Appl. Opt. 20, 1333 (1981).

[7] R. W. Dixon, J. Appl. Phys. 38, 5149 (1967).

[8] D. A. Pinnow, IEEE J. Quantum Electron. 6, 223 (1970).

[9] A. Korpel, in Applied Solid State Physics, edited by R. Wolfe, (Academic, New York, 1972), Vol. 3, pp. 71-180.

[10] E. G. Spencer, P. V. Lenzo, and A. A. Ballman, Proc. IEEE 55, 2074 (1967).

[11] T. C. Lee and J. D. Zook, IEEE J. Quantum Electron. 4, 442 (1968).

[12] J. F. Lotspeich, IEEE Spectrum 5, 45 (1968).

[13] J. D. Zook, Appl. Opt. 13, 875 (1974).

[14] H. Kosaka, T. Kawashima, A. Tomita, M. Notomi, T. Tamamura, T. Sato, and S. Kawakami, Phys. Rev. B 58, R10096 (1998).

[15] T. Baba and M. Nakamura, IEEE J. Quantum Electron. 38, 909 (2002).

[16] F. Vasey, F. K. Reinhart, R. Houdre, and J. M. Stauffer, Appl. Opt. 32, 3220 (1993).

[17] M. W. Farn, Appl. Opt. 33, 5151 (1994).

[18] S. Ahderom, M. Raisi, K. Lo, K. E. Alameh, and R. Mavaddat, in 5th IEEE International Conference on High-Speed Networks and Multimedia Communications (Edith Cowan University, Joondalup, Australia, 2002), pp. 239-242.

[19] P. F. McManamon, Opt. Photonics News 17, No. 3, 24 (2006)

[20] A. C. Tam and W. Happer, Phys. Rev. Lett. 38, 278 (1977).

[21] G. T. Purves, G. Jundt, C. S. Adams, and I. G. Hughes, Eur. Phys. J. D 29, 433 (2004).

[22] S. E. Harris, J. E. Field, and A. Imamoglu, Phys. Rev. Lett. 64, 1107 (1990).
[23] M. O. Scully and S. Y. Zhu, Opt. Commun. 87, 134 (1992).

[24] M. Xiao, Y. Q. Li, S. Z. Jin, and J. Gea-Banacloche, Phys. Rev. Lett. 74, 666 (1995).

[25] R. R. Moseley, S. Shepherd, D. J. Fulton, B. D. Sinclair, and M. H. Dunn, Phys. Rev. Lett. 74, 670 (1995).

[26] R. R. Moseley, S. Shepherd, D. J. Fulton, B. D. Sinclair, and M. H. Dunn, Phys. Rev. A 53, 408 (1996).

[27] M. Mitsunaga, M. Yamashita, and H. Inoue, Phys. Rev. A 62, 013817 (2000).

[28] A. G. Truscott, M. E. J. Friese, N. R. Heckenberg, and H. Rubinsztein-Dunlop, Phys. Rev. Lett. 82, 1438 (1999).

[29] J. A. Andersen, M. E. J. Friese, A. G. Truscott, Z. Ficek, P. D. Drummond, N. R. Heckenberg, and H. Rubinsztein-Dunlop, Phys. Rev. A 63, 023820 (2001).

[30] R. Kapoor and G. S. Agarwal, Phys. Rev. A 61, 053818 (2000).

[31] D. R. Walker, D. D. Yavuz, M. Y. Shverdin, G. Y. Yin, A. V. Sokolov, and S. E. Harris, Opt. Lett. 27, 2094 (2002).

[32] D. D. Yavuz, D. R. Walker, and M. Y. Shverdin, Phys. Rev. A 67, 041803(R) (2003).

[33] M. Y. Shverdin, D. D. Yavuz, and D. R. Walker, Phys. Rev. A 69, 031801(R) (2004).

[34] J. T. Manassah and B. Gross, Opt. Commun. 124, 418 (1996).

[35] I. V. Kazinets, B. G. Matisov, and A. Y. Snegirev, Tech. Phys. 42, 837 (1997).

[36] H. Shpaisman, A. D. Wilson-Gordon, and H. Friedmann, Phys. Rev. A 71, 043812 (2005).

[37] M. Born and E. Wolf, Principles of Optics: Electromagnetic Theory of Propagation, Interference and Diffraction of Light, 7th ed. (Cambridge University Press, Cambridge, U.K., 1999).

[38] M. O. Scully and M. S. Zubairy, Quantum Optics (Cambridge University Press, Cambridge, 1997).

[39] A. Y. Savchencko and B. Y. Zel'dovich, J. Opt. Soc. Am. B 13, 273 (1996).

[40] A. Ciattoni, P. D. Porto, B. Crosignani, and A. Yariv, J. Opt. Soc. Am. B 17, 809 (2000).

[41] Q. Sun, Y. V. Rostovtsev, J. P. Dowling, M. O. Scully, and M. S. Zubairy, Phys. Rev. A 72, 031802(R) (2005). 